\title{
Predicting Blood Flow Responses to Rhythmic Handgrip Exercise From One Second Isometric Contractions
}

\author{
M. COOK ${ }^{1}$, N. A. SMART ${ }^{1}$, T. VAN DER TOUW ${ }^{1}$ \\ ${ }^{1}$ School of Science and Technology, University of New England, Armidale, Australia
}

Received September 17, 2015

Accepted December 4, 2015

On-line March 15, 2016

\section{Summary}

The aim of this work was to predict blood flow responses to rhythmic handgrip exercise from one second isometric contractions. Seven healthy men were studied. Each subject performed a single $1 \mathrm{~s}$ handgrip contraction at $10 \%, 20 \%$ and $40 \%$ of the maximum handgrip strength. We then repeatedly summed hyperaemic responses from single contractions to predict hyperaemic response to a prolonged bout of rhythmic exercise. There was similarity between steady state brachial blood flow velocity (BBV) extrapolated from single handgrip contractions and during 2 min of rhythmic exercise for $20 \%$ $(10.0 \pm 3.8 \mathrm{~cm} / \mathrm{s}$ vs. $10.2 \pm 2.6 \mathrm{~cm} / \mathrm{s}, \mathrm{r}=0.93, \mathrm{p}=0.003)$ and $40 \%$ of maximum contractions $(14.2 \pm 5.5 \mathrm{~cm} / \mathrm{s}$ vs. $15.6 \pm 3.4 \mathrm{~cm} / \mathrm{s}$, $r=0.88, p=0.009)$, but not for $10 \%(7.5 \pm 4.1 \mathrm{~cm} / \mathrm{s}$ vs. $5.7 \pm$ $3.3 \mathrm{~cm} / \mathrm{s}, \mathrm{r}=0.94, \mathrm{p}=0.018$ ). BBV progressively rose substantially higher during rhythmic contractions than peak BBV observed during single contractions at matched intensity. Respective peak BBV during single contractions and steady state BBV rhythmic contractions were $4.4 \pm 2.1$ and $5.7 \pm 3.3 \mathrm{~cm} . \mathrm{s}^{-1}$ at $10 \%$ forearm strength $(p=0.14), 5.6 \pm 2.4$ and $10.2 \pm 2.8 \mathrm{~cm} . \mathrm{s}^{-1}$ at $20 \%$ $(p=0.002)$, and $7.0 \pm 2.5$ and $15.6 \pm 3.6 \mathrm{~cm} . \mathrm{s}^{-1}$ at $40 \%$ $(p=0.003)$. In conclusion, there is similarity between the summated blood flow velocity calculated from a single $1 \mathrm{~s}$ muscle contraction and the steady state blood flow velocity response of rhythmic exercise.

\section{Key words}

Brachial blood flow • Isometric exercise - Hyperaemia • Blood flow control

\section{Corresponding author}

N. A. Smart, School of Science and Technology, University of New England, Armidale, NSW 2351, Australia. Fax: +61-26773-5011. E-mail: Nsmart2@une.edu.au

\section{Introduction}

Haemodynamic responses to isometric resistance exercise and remote ischaemic pre-conditioning have attracted attention as potential therapeutic interventions for a range of clinical disorders including hypertension (Carlson et al. 2014) and wound healing (Shaked et al. 2015). The mechanism(s) responsible for these improvements remain unclear, but ischaemia is considered to be the principal initiating stimulus (Helgeland et al. 2014). The intensity and duration of the ischaemia which yields optimal haemodynamic benefits remain to be determined, as does the most effective manner to induce ischaemia.

Exercise involving sustained isometric handgrip contractions has been shown to be an effective means of reducing blood pressure (Cornelissen et al. 2013, Cornelissen and Smart 2013, Inder et al. 2016), but can lead to muscle fatigue or discomfort. Such discomfort could be reduced by adopting rhythmic handgrip contractions interspersed with brief intervening periods of relaxation. However, the efficacy of rhythmic handgrip contractions in inducing therapeutic haemodynamic improvements has not been studied, and it is unknown whether sustained or rhythmic contractions are superior. On the one hand, sustained contractions could induce a more intense level of ischaemia over a given period of time than would rhythmic contractions of comparable strength. However, rhythmic contractions can be sustained for longer periods than sustained contractions, and may therefore impose a more prolonged ischaemic challenge than would be possible with sustained contractions of comparable strength. 
Recent work on two therapeutic techniques used to induce muscle ischaemia, isometric resistance exercise and remote ischaemic pre-conditioning, has shown chronic improvements in wound healing and blood pressure with repeated exposure (Carlson et al. 2014, Cornelissen et al. 2013, Shaked et al. 2015). While the mechanisms that regulate the magnitude and timing of muscle ischaemia during exercise have not been fully elucidated, an enhanced understanding of such mechanisms could help identify the most effective manner to therapeutically induce haemodynamic benefits from repeated exposure to ischaemic interventions. A key feature which determines how effectively rhythmic muscle contractions induce muscle ischaemia is the degree of recovery that occurs between successive contractions. The hyperaemic response from a single brief muscle contraction commences within the first second after cessation of the contraction, but recovery is not immediate as evidenced by the persistence of hyperaemia for some time after cessation of the contraction (Cornelissen and Smart 2013, Hughson 2003, Inder et al. 2016, Joyner and Casey 2014). Periods of relaxation that are too brief for complete recovery to occur between successive contractions could therefore potentially result in the development of progressively increasing levels of ischaemia and hyperaemia over time with subsequent contractions. If this framework is correct, then it could theoretically be possible to predict the hyperaemic response to repeated rhythmic contractions by summating the hyperaemic response to a single contraction. To explore this possibility we compared the magnitude and time course of the hyperaemic response to handgrip exercise by comparing the hyperaemic response to 2 min of rhythmic handgrip contractions with the theoretical hyperaemic response calculated by repeatedly summating the hyperaemic response from a single brief handgrip contraction over a $2 \mathrm{~min}$ period. The purpose of this study was to provide an enhanced understanding of the hyperaemic and ischaemic responses to muscle contractions to inform the development of an effective therapeutic intervention for the management of clinical disorders such as hypertension.

\section{Methods}

Seven healthy non-smoking, recreationally trained, men, between 20 to 23 years of age, unmedicated, with no known disease were studied, mean age $21.4 \pm 0.9$ (SD) years, height $182.7 \pm 6.0$ and body mass $87.3 \pm 16.6 \mathrm{~kg}$. The study was approved by the Human Research Ethics Committee at the University of New England and all subjects gave their informed consent.

\section{Equipment}

Brachial arterial blood flow velocity (BBV) was measured with a $5 \mathrm{MHz}$ transcutaneous ultrasound Doppler probe (Hokanson Bidirectional Doppler MD6, SDR Clinical Technology, Middle Cove, Australia) placed over the brachial artery in the antecubital fossa of the subject's dominant arm. The Doppler probe was placed at a $60^{\circ}$ degree angle relative to the forearm and was securely held in place with a purpose built housing constructed from compacted foam. Voluntary isometric handgrip contraction force generated by the finger flexor muscles was recorded from the dominant forearm using a purpose built handgrip ergonometer that provided an electrical output. Electrical outputs from the Doppler probe and ergonometer were recorded on a personal computer data acquisition system (PowerLab model no. ML796, ADInstruments, Sydney) and dedicated software (Chart v5.5.4, ADInstruments, Sydney) at a sampling rate of $1 \mathrm{kHz}$. A computer digital metronome program (WEIRD METRONOME v1.2, Los Angeles, CA) was used throughout each study to provide handgrip contraction and relaxation timing cues to the subjects.

\section{Protocol}

All subjects were studied in the seated position with their dominant arm outstretched horizontally at level of the heart and the elbow fully extended. The height of the laboratory equipment and the subject's seat was adjusted to accommodate each subject's individual body size. Preliminary measurements were made on each subject to ensure that an adequate and reproducible BBV signal could be recorded, and to ensure that the subjects were physically able to repeatedly maintain rhythmic handgrip contractions at preset levels of force for $2 \mathrm{~min}$ periods.

To standardize against inter-subject differences in handgrip strength, handgrip contraction force for each subject was expressed in terms of the maximum handgrip force that could be generated. To determine the dominant forearm's handgrip strength, each subject performed brief (1 s duration) maximum handgrip contractions until 3 contractions were obtained that each had a peak force that agreed within $\pm 10 \%$ of each other. Maximum handgrip strength was defined as the average of the peak 
force from these 3 contractions. $10 \%, 20 \%$ and $40 \%$ of the maximum handgrip strength was then calculated for each subject.

After ensuring that resting $\mathrm{BBV}$ remained constant for at least $5 \mathrm{~min}$, each subject performed a single $1 \mathrm{~s}$ handgrip contraction at $10 \%$ of the maximum handgrip strength. Subjects then rested until BBV returned to baseline. This process was repeated until four $\mathrm{BBV}$ responses at $10 \%$ of the maximum handgrip strength had been recorded. This procedure was repeated for handgrip contractions at $20 \%$, and then $40 \%$, of the maximum handgrip strength.

After ensuring that resting BBV remained constant for at least $5 \mathrm{~min}$, each subject performed forearm exercise consisting of $2 \mathrm{~min}$ of rhythmic handgrip contractions with each contraction at $10 \%$ of the maximum handgrip strength lasting $1 \mathrm{~s}$ and with a $2 \mathrm{~s}$ relaxation period between successive contractions. Subjects then rested until BBV returned to its resting level. The 2 min rhythmic contraction protocol was repeated if examination of the $\mathrm{BBV}$ and force recordings revealed that the protocol had not been adequately adhered to, or the equipment had not performed satisfactorily. This procedure was repeated for $2 \mathrm{~min}$ of rhythmic handgrip contractions at $20 \%$ of maximum handgrip strength, and at $40 \%$ of maximum handgrip strength. Throughout each $2 \mathrm{~min}$ period of rhythmic handgrip contractions each subject received continuous feedback of their handgrip contraction force by viewing the handgrip force-time signal on the computer screen.

\section{Data analysis}

BBV signals were recorded on Chart software in millivolts $(\mathrm{mV})$, and were then expressed in centimeters per second $(\mathrm{cm} / \mathrm{s})$ using equation 1 as recommended by the Doppler probe manufacturers (Hokanson, D. E. Inc., Middle Cove, Australia, 2002).

$$
\begin{aligned}
& \text { Blood flow velocity }(\mathrm{cm} / \mathrm{s})= \\
& =\frac{\text { Doppler probe electronic output }(\mathrm{mV}) \times 10}{64.9 \times \cos 60^{\circ} \times 6}
\end{aligned}
$$

Equation 1

Using data analysis functions available on Chart software, resting BBV was averaged for the two cardiac cycles immediately prior to individual $1 \mathrm{~s}$ handgrip contractions. BBV was also averaged for each consecutive cardiac cycle after individual $1 \mathrm{~s}$ handgrip contractions until BBV returned to resting levels. All cardiac cycle averaged BBV data was then expressed as the change from resting levels $(\triangle \mathrm{BBV})$. A third order multinomial regression equation was calculated for the $\triangle \mathrm{BBV}$ and corresponding time data after each individual handgrip contraction. The multinomial regression equation was used to calculate the predicted $\triangle \mathrm{BBV}$ for each second after the single handgrip contraction $\left(\triangle \mathrm{BBV}_{\text {predicted }}\right)$ until blood flow velocity returned to the resting level. This mathematical analysis provided 4 sets of $\triangle \mathrm{BBV}_{\text {predicted }}$ and corresponding time data for each handgrip contraction intensity in each subject. Data were then averaged to yield one averaged set of $\triangle \mathrm{BBV}_{\text {predicted }}$ data for each contraction intensity in each subject. Each averaged set of $\triangle \mathrm{BBV}_{\text {predicted }}$ data was then added to itself with a $3 \mathrm{~s}$ time delay to calculate the predicted $\triangle \mathrm{BBV}_{\text {predicted }}$ data set corresponding to two $1 \mathrm{~s}$ handgrip contractions interspersed with a $2 \mathrm{~s}$ period of relaxation. The additive process was repeated a total of 40 times, each time extending the time delay by a further $3 \mathrm{~s}$, to yield summated data $\left(\sum \Delta \mathrm{BBV}_{\text {predicted }}\right)$ that corresponded to the change in cardiac cycle averaged BBV data during each second over a 2 min period of rhythmic exercise consisting of forty $1 \mathrm{~s}$ handgrip contractions that were each interspersed with a $2 \mathrm{~s}$ period of relaxation. After summation, only $\sum \Delta \mathrm{BBV}_{\text {predicted }}$ data from every $3^{\text {rd }}$ second were retained to facilitate comparison with corresponding data measured over the first cardiac cycle that occurred after each handgrip contraction during 2 min periods of rhythmic handgrip contractions (see below). This analysis was performed separately in each subject for each of the 3 contraction intensities studied to yield 3 plots for each subject showing $\Sigma \Delta \mathrm{BBV}_{\text {predicted }}$ versus time during a $2 \mathrm{~min}$ period of rhythmic handgrip contractions.

Resting BBV was averaged for two cardiac cycles immediately prior to $2 \mathrm{~min}$ of rhythmic handgrip contractions. BBV was also averaged over the first cardiac cycle after handgrip contraction ceased during each 2 s relaxation period during the 2 min rhythmic contraction period. The changes in cardiac cycle averaged BBV from resting levels measured during the $2 \mathrm{~min}$ period of rhythmic handgrip contractions $\left(\triangle \mathrm{BBV}_{\text {measured }}\right)$ was then plotted against the corresponding time data. This analysis was performed separately for each of the 3 contraction intensities that were studied to yield 3 plots for each subject showing $\triangle \mathrm{BBV}_{\text {measured }}$ versus time during the 2 min period of rhythmic handgrip contractions.

Unless otherwise stated, group data are expressed as means and standard deviations (SD). Paired 
comparisons were performed with the 2 tailed paired Student's t test, with the null hypothesis rejected at $\mathrm{p}<0.05$. One way ANOVA was used for multiple paired comparisons, but with the null hypothesis rejected at $\mathrm{p}<0.017$ to accommodate Bonferroni's adjustments for 3 inter-comparisons. Pearson correlation coefficients were used to compare the similarity between steady state and rhythmic exercise.

\section{Results}

Seven subjects were studied after fulfilling the brachial arterial blood flow velocity (BBV) and muscle contraction screening criteria. Each subject was able to generate the required level of handgrip force throughout the 2 min periods of rhythmic contraction. Resting BBV prior to the 3 levels of contraction intensity not differ with handgrip intensity (all NS), (10\%) $4.7 \pm 2.2 \mathrm{~cm} . \mathrm{s}^{-1}$; (20\%) $5.1 \pm 3.3 \mathrm{~cm} . \mathrm{s}^{-1}$; (40\%) $5.8 \pm 4.1 \mathrm{~cm} . \mathrm{s}^{-1}$.

Figures 1a-1c show predicted blood flow velocity responses to repeated forearm contractions at $10 \%, 20 \%$ and $40 \%$ of forearm strength. The predicted blood flow velocity responses were calculated by repeatedly summating the blood flow velocity responses to a single $1 \mathrm{~s}$ forearm contraction. a

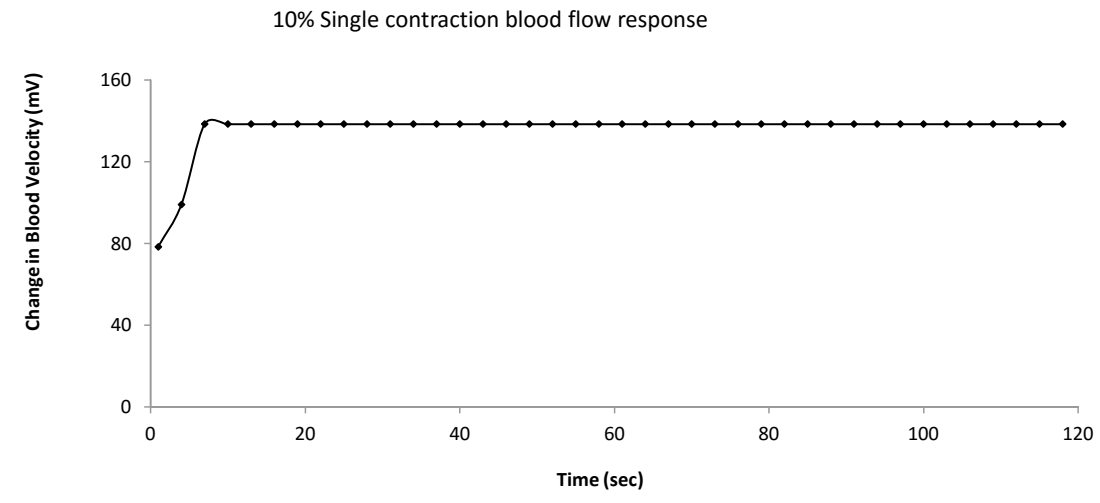

b

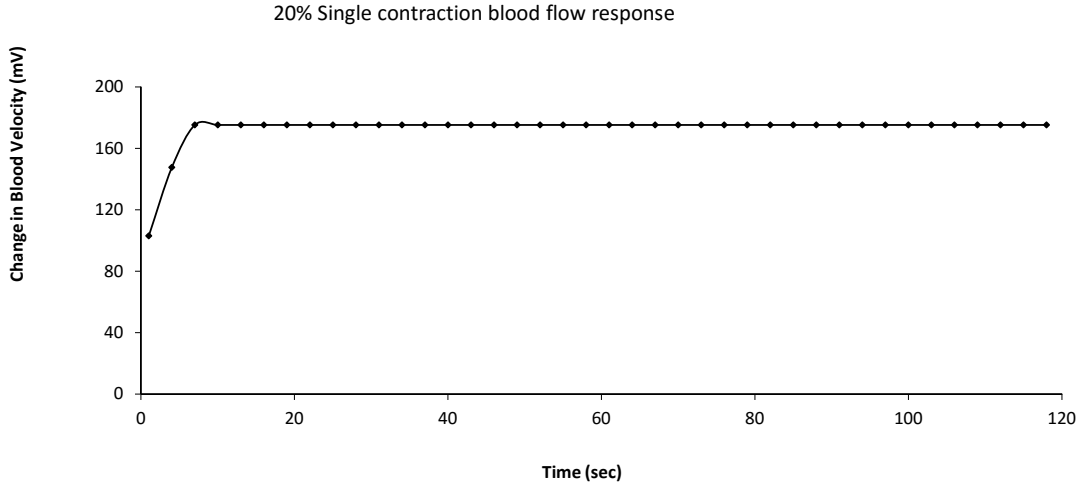

c

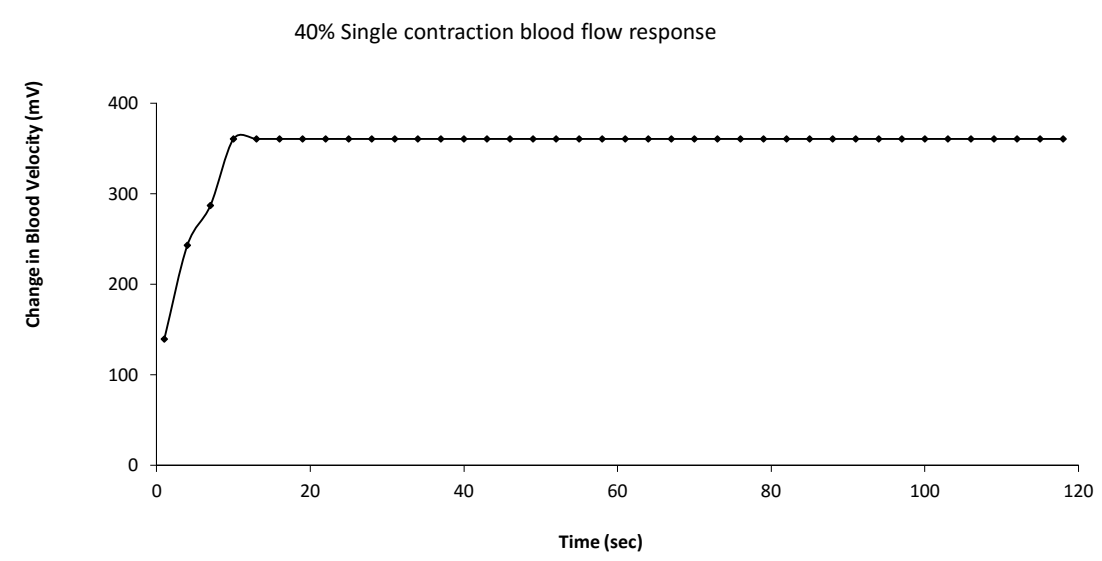

Fig. 1. Predicted blood flow velocity response to repeated forearm contractions at (a) $10 \%$, (b) $20 \%$ and (c) $40 \%$ of forearm strength (subject 6). 


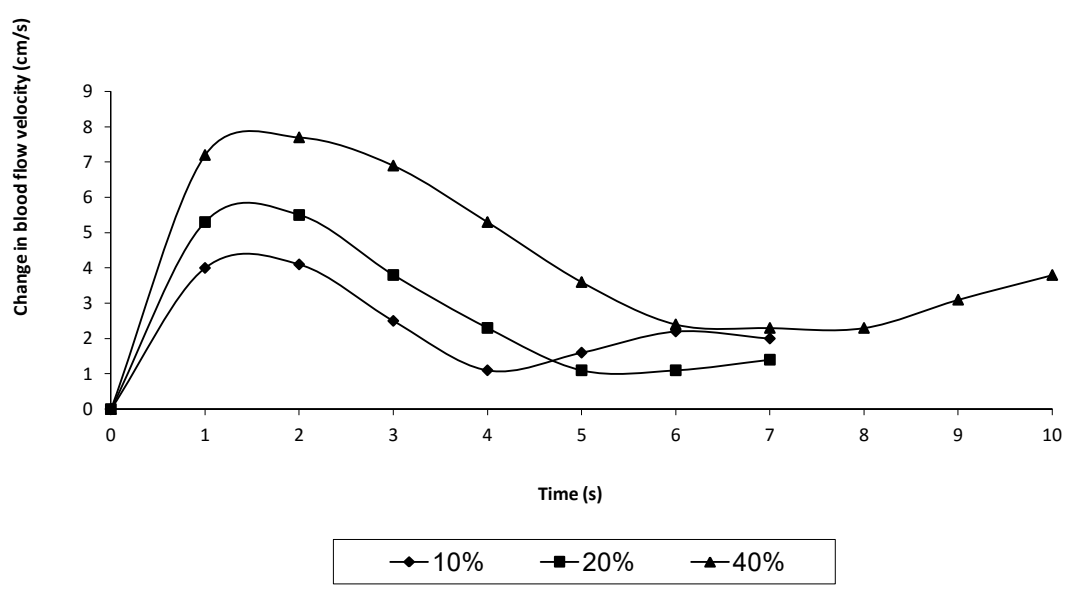

Fig. 2. Representative blood flow velocity responses to individual $1 \mathrm{~s}$ forearm contractions (subject 6).

\section{$B B V$ responses to single contractions}

BBV increased rapidly after a single handgrip contraction and then steadily decreased to return to baseline within $12 \mathrm{~s}$ (Fig. 2). The peak values of the BBV response to single contractions progressively increased with increasing contraction intensity and equalled $4.4 \pm$ $1.9 \mathrm{~cm} . \mathrm{s}^{-1}$ at $10 \%$ of forearm strength, $5.6 \pm 2.2 \mathrm{~cm} . \mathrm{s}^{-1}$ at $20 \%$ of forearm strength, and $7.0 \pm 2.3 \mathrm{~cm} . \mathrm{s}^{-1}$ at $40 \%$ of forearm strength, although only the $10 \%$ and $40 \%$ groups differed significantly $(\mathrm{p} \leq 0.017)$. The duration of the $\mathrm{BBV}$ response represents the period of time in seconds the BBV contraction response lasted after cessation of a single contraction and was $8.0 \pm 1.7 \mathrm{~s}$ at $10 \%$ of forearm strength, $9.0 \pm 2.7 \mathrm{~s}$ at $20 \%$ of forearm strength, and $9.7 \pm 2.4 \mathrm{~s}$ at $40 \%$ of forearm strength, all $\mathrm{p}$ between groups was $\geq 0.017$. The null hypothesis was rejected at $\mathrm{p}<0.017$ to accommodate Bonferroni's adjustments for comparisons between the 3 contraction intensities, although a borderline difference $(p=0.047)$ was observed between the durations of the responses to the $10 \%$ and $40 \%$ maximum contractions.

Table 1. Comparison of times taken for single and continuous contraction curves to reach $80 \%$ of their respective maximums at different contraction intensities.

\begin{tabular}{|c|c|c|c|c|c|c|}
\hline \multirow[b]{2}{*}{ Subject } & \multicolumn{2}{|c|}{$10 \% \max$} & \multicolumn{2}{|c|}{$20 \% \max$} & \multicolumn{2}{|c|}{$40 \% \max$} \\
\hline & Single & Continuous & Single & Continuous & Single & Continuous \\
\hline 1 & 25 & 41.1 & 5 & 29 & 5 & 8.8 \\
\hline 2 & 25 & 53.3 & 16 & 37.2 & 7 & 9.8 \\
\hline 3 & 4 & 28.8 & 7 & 83.3 & 11 & 41.2 \\
\hline 4 & 25 & 49.4 & 28 & 21.3 & 11 & 44.6 \\
\hline 5 & 14 & 29.6 & 7 & 101.7 & 13 & 24.8 \\
\hline 6 & 7 & 25.7 & 4 & 52.3 & 10 & 37.3 \\
\hline 7 & 4 & 32.9 & 7 & 68.3 & 4 & 18 \\
\hline Mean & 14.9 & 37.3 & 10.6 & 56.2 & 8.7 & 26.4 \\
\hline$S D$ & 9.3 & 10.0 & 8.0 & 27.5 & 3.2 & 13.7 \\
\hline$p$ & \multicolumn{2}{|c|}{0.00001} & \multicolumn{2}{|c|}{0.014} & \multicolumn{2}{|c|}{0.010} \\
\hline
\end{tabular}

Numeric data are time values measured in seconds. $10 \% \max , 20 \% \max$ and $40 \%$ max refer to forearm muscle contractions at 10,20 and $40 \%$ of the subjects' maximal contraction strength. Single refers to the predicted blood flow velocity which was extrapolated from a single one second forearm contraction. Continuous refers to the blood flow velocity measured during rhythmic forearm contractions.

Calculated and measured BBV responses to rhythmic contractions

There was good agreement (88-94\% accuracy) between the actual steady state blood flow responses and those that were predicted by extrapolating from a single handgrip contraction and values during 2 min of rhythmic exercise. These values were significant for $20 \%$ $\left(10.0 \pm 3.8 \mathrm{~cm} . \mathrm{s}^{-1}\right.$ vs. $\left.10.2 \pm 2.6 \mathrm{~cm} . \mathrm{s}^{-1}, \mathrm{r}=0.93, \mathrm{p}=0.003\right)$ 
and $40 \%$ of maximum contractions $\left(14.2 \pm 5.5 \mathrm{~cm} . \mathrm{s}^{-1}\right.$ vs. $\left.15.6 \pm 3.4 \mathrm{~cm} . \mathrm{s}^{-1}, \mathrm{r}=0.88, \mathrm{p}=0.009\right)$, but not for $10 \%$ $\left(7.5 \pm 4.1 \mathrm{~cm} . \mathrm{s}^{-1}\right.$ vs. $\left.5.7 \pm 3.3 \mathrm{~cm} . \mathrm{s}^{-1}, \mathrm{r}=0.94, \mathrm{p}=0.018\right)$ (a representative example for one subject is shown in Fig. 3). The hyperaemic response measured during 2 min of repeated handgrip contractions lagged behind that predicted on the basis of a single handgrip contraction, and this was evident when all subjects achieved at least $80 \%$ of their maximum BBV responses (Table 1). Although this effect was evident for each of the 3 workloads studied, the magnitude of the time difference did not appear to be workload dependent.

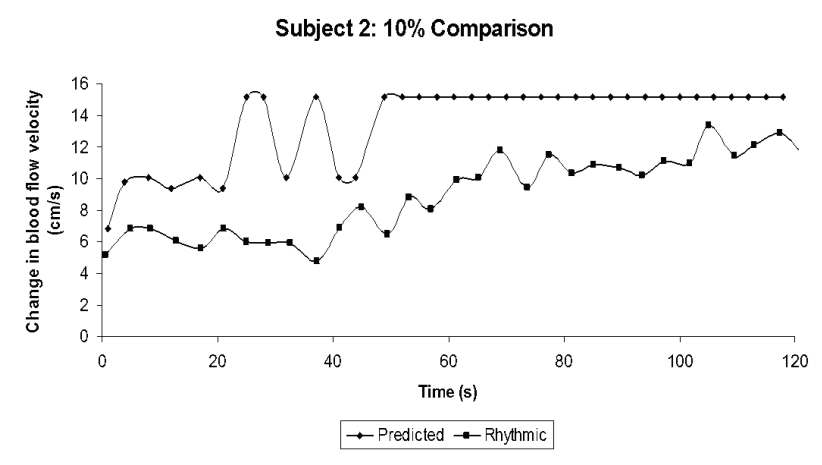

Subject 2: 20\% Comparison

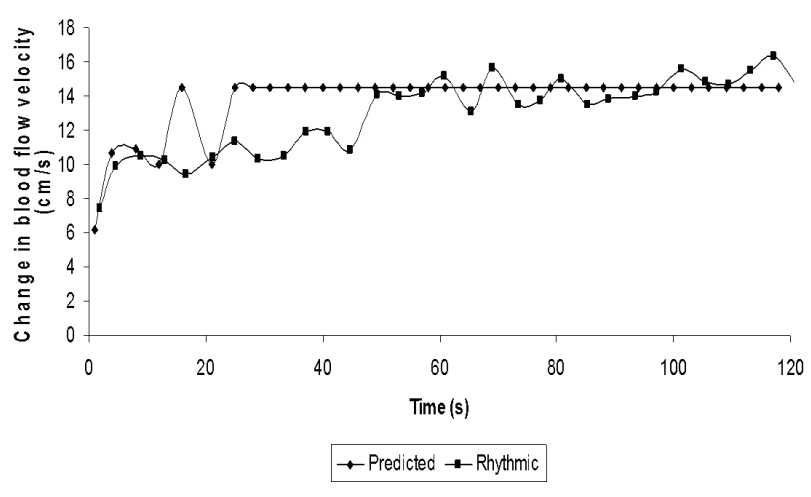

Subject 2: $40 \%$ Comparison

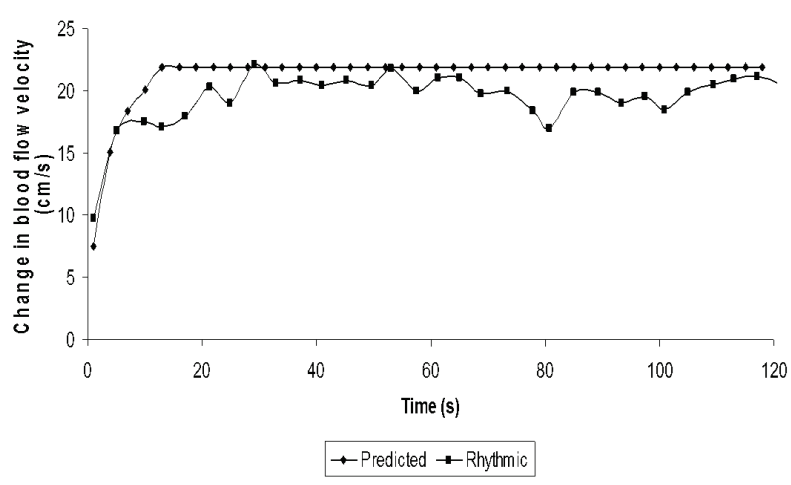

Fig. 3. Predicted and actual blood flow velocity responses for 2 min of rhythmic forearm contractions at 3 levels of contraction force in one subject.
Peak BBV responses during single and rhythmic contractions

BBV progressively rose to substantially higher levels during rhythmic contractions than the peak values observed during single contractions at matched levels of forearm contraction. This effect became increasingly evident at higher levels of contraction with peak BBV during single contractions and steady state BBV during 2 min of rhythmic contractions respectively equalling $4.4 \pm 2.1$ and $5.7 \pm 3.3 \mathrm{~cm} . \mathrm{s}^{-1}$ at $10 \%$ of forearm strength $(\mathrm{p}=0.14), 5.6 \pm 2.4$ and $10.2 \pm 2.8 \mathrm{~cm} . \mathrm{s}^{-1}$ at $20 \%$ of forearm strength $(\mathrm{p}=0.002)$, and $7.0 \pm 2.5$ and $15.6 \pm 3.6 \mathrm{~cm} . \mathrm{s}^{-1}$ at $40 \%$ of forearm strength $(\mathrm{p}=0.003)$.

\section{Discussion}

This is the first study to demonstrate that the steady state hyperaemic response of the human forearm undergoing exercise consisting of rhythmic contractions can be predicted from the hyperaemic response to a single brief forearm contraction. The summated blood flow velocity calculated from a single $1 \mathrm{~s}$ muscle contraction accurately predicted the steady state blood flow velocity response of rhythmic exercise. The results of this study reveal that the failure to achieve full recovery from a single brief muscle contraction before the commencement of subsequent contractions results in the development of progressively increasing levels of hyperaemia over time during rhythmic contractions. This finding suggests that rhythmic contractions can induce high levels of muscle ischaemia over a sustained period which could be of use as a potential therapeutic intervention for a range of clinical disorders including hypertension. Our work raises significant questions as to how the body is able to rapidly adjust its blood flow to precisely match the required increase in metabolic demand. The hyperaemic response to exercise predicted on the basis of summated single brief muscle contractions was found to achieve steady state more rapidly than that directly measured during 2 min of rhythmic contractions, and this finding provides further insights into the mechanisms regulating the immediate, and the slower onset steady state, hyperaemic responses to exercise.

Brachial arterial blood flow velocity progressively rose to substantially higher levels during rhythmic contractions than the peak values observed during single contractions at matched levels of forearm contraction. This effect became increasingly evident at higher levels of contraction. These findings suggest that 
rhythmic contractions can induce high levels of muscle ischaemia over a sustained period so that rhythmic contractions may be of use as a potential therapeutic intervention for a range of clinical disorders including hypertension.

The mechanisms which determine the magnitude and time course of the hyperaemic response to exercise have not been fully elucidated. Several minutes are required after the onset of exercise before physiological mechanisms adjust to allow aerobic processes to fully meet the elevated metabolic needs of the participating muscles (Hughson 2003). An important component of this adjustment is the increase in blood flow to meet the increased metabolic demand of the participating skeletal muscles (Joyner and Casey 2014). Studies examining the muscle hyperaemic response to exercise have identified a biphasic pattern consisting of a rapid initial vasodilation (Phase I) which is followed by a slower Phase II vasodilation. Although considerable variation in the time course of these events has been reported, the Phase I response frequently peaks around 5 to $7 \mathrm{~s}$ after the onset of exercise, plateaus for 10 to $15 \mathrm{~s}$, and then progressively rises further at a slower rate (Phase II) until steady-state levels are achieved (Shoemaker et al. 1998). Similar work (Rogers et al. 2006), like ours, have employed voluntary rhythmic handgrip contractions for reasons of laboratory convenience, thereby allowing direct comparison between the time course data of the present study and the published literature.

The mechanisms responsible for the rapid onset and the later onset hyperaemic responses to exercise remain unclear (MacDonald et al. 1998) and complex exercise protocols with varying loads and contraction rates have been utilized to explore this issue (Rogers et al. 2006). In the present study, the hyperaemic response to single handgrip contractions was rapid in onset (within 1-2 $\mathrm{s}$ after the contraction), peaked within 2-5 s after the contraction, and dissipated within 6-15 s after the single contraction. In addition, the peak blood flow velocity response to a single handgrip contraction was directly related to the contraction force, which implies that there is a direct stimulus-response relationship between contractile force and the blood flow response. These findings are consistent with previous work (Shoemaker and Hughson 1999). The rapid time course of this response appears inconsistent with humoral feedback mechanisms and appears more consistent with a neurally mediated response. However, the hyperaemic response measured during 2 min of repeated handgrip contractions lagged behind that predicted on the basis of a single handgrip contraction (Table 1). This suggests that the initial hyperaemic response to a single contraction diminishes somewhat with subsequent contractions, and that other hyperaemic processes progressively come into play during repeated contractions until a steady state hyperaemic response is achieved. Such a rapid onset and adaptive diminishing response is a feature of many neurally mediated control mechanisms, including those elicited during exercise (Turner 1991). Moreover, a rapid onset and adaptive initial hyperaemic response, followed by other slower onset hyperaemic processes, could account for the Phase I and Phase II hyperaemic responses observed in previous work (Shoemaker et al. 1998).

A principal finding of this study was that the human forearm vasculature can precisely match the required increase in blood flow to meet metabolic demand within seconds after a single $1 \mathrm{~s}$ handgrip contraction. The speed with which the human forearm vasculature can precisely match the required increase in blood flow to meet metabolic demand during single muscle contractions suggests the involvement of mechanisms not dependent on metabolic feedback.

\section{Critique of methods}

A key issue in this study was the subjects' compliance in carrying out the detailed requirements of the experimental protocol of this experiment. To optimize subject compliance, each subject underwent several practice sessions to familiarize themselves with the protocols before the experiment. Post study examination of the forearm contraction force-time profiles from each subject revealed a high level of compliance, with all subjects maintaining handgrip contractions within $\pm 10 \%$ of the preset force throughout the required duration for each handgrip contraction. Serial measurements of brachial arterial internal diameter were not measured in this study. Therefore, it is possible that changes in BBV did not accurately reflect changes in brachial arterial blood flow. However, the findings by Rogers et al. (2006) argue against this possibility, with essentially no change in brachial arterial diameter observed during periods of rhythmic forearm contractions.

BBV transiently dropped to low levels at times. It is not known whether such fluctuations were the result of a movement artefact. However, such large transient changes appear non-physiological, and did not influence the overall findings of this study. 
The protocol of this study utilized weak handgrip contractions first, and then consistently progressed to more forceful contractions. However, the different contraction force studies were clearly interspersed with adequate rest times, as evidenced by the lack of change in resting $\mathrm{BBV}$ prior to the commencement of the 3 levels of contraction force. In addition, these data are consistent with that from previously reported studies (Tschakovsky et al. 1996).

\section{Conclusions}

The summated blood flow velocity calculated from a single $1 \mathrm{~s}$ muscle contraction was found to predict, with $>88 \%$ accuracy, the steady state blood flow velocity response of rhythmic exercise. This study further demonstrated the development of progressively increasing levels of hyperaemia over time during rhythmic forearm contractions when there was only partial recovery from a single brief muscle contraction before the commencement of subsequent contractions.
Rhythmic contractions may therefore be capable of inducing high levels of muscle ischaemia over a sustained period, and such a response could potentially be of use therapeutically to manage a range of clinical disorders including hypertension. The speed with which the human forearm vasculature can precisely match the required increase in blood flow to meet metabolic demand during single muscle contractions suggests the involvement of mechanisms not dependent on metabolic feedback. In addition, the results of this study confirm previous reports that the rapid onset hyperaemic response to single muscle contractions is dependent on the force of the contraction.

\section{Conflict of Interest}

There is no conflict of interest.

\section{Acknowledgements}

The authors express their appreciation to Dr. Simon Green and Ms Candice Savell for assistance in constructing the Doppler probe housing.

\section{References}

CARLSON DJ, DIEBERG G, HESS NCL, MILLAR PJ, SMART NA: Isometric exercise training for blood pressure management: a systematic review and meta-analysis. Mayo Clin Proc 89: 327-334, 2014.

CORNELISSEN VA, SMART NA: Exercise training for blood pressure: a systematic review and meta-analysis. $J$ Am Heart Assoc 2: e004473, 2013.

CORNELISSEN VA, BUYS R, SMART NA: Endurance exercise beneficially affects ambulatory blood pressure: a systematic review and meta-analysis. J Hypertens 31: 639-648, 2013.

HELGELAND E, BREIVIK LE, VAUDEL M, SVENDSEN OS, GARBERG H, NORDREHAUG JE, BERVEN FS, JONASSEN AK: Exploring the human plasma proteome for humoral mediators of remote ischemic preconditioning - a word of caution. PLoS One 9: e109279, 2014. doi: 10.1371/journal.pone.0109279

HUGHSON RL: Regulation of blood flow at the onset of exercise by feed forward and feedback mechanisms. Can J Appl Physiol 28: 774-787, 2003.

INDER JD, CARLSON DJ, DIEBERG G, MCFARLANE JR, HESS NCL, SMART NA: Isometric exercise training for blood pressure management: a systematic review and meta-analysis to optimize benefit. Hypertens Res 39: 88-94, 2016.

JOYNER MJ, CASEY DP: Muscle blood flow, hypoxia, and hypoperfusion. J Appl Physiol 116: 852-857, 2014.

MACDONALD MJ, SHOEMAKER JK, TSCHAKOVSKY ME, HUGHSON RL: Alveolar oxygen uptake and femoral artery blood flow dynamics in upright and supine leg exercise in humans. J Appl Physiol 85: 1622-1628, 1998.

ROGERS AM, SAUNDERS NR, PYKE KE, TSCHAKOVSKY ME: Rapid vasoregulatory mechanisms in exercising human skeletal muscle: dynamic response to repeated changes in contraction intensity. Am J Physiol Heart Circ Physiol 291: H1065-H1073, 2006.

SHAKED G, CZEIGER D, ABU ARAR A, KATZ T, HARMAN-BOEHM I, SEBBAG G: Intermittent cycles of remote ischemic preconditioning augment diabetic foot ulcer healing. Wound Repair Regen 23: 191-196, 2015.

SHOEMAKER JK, HUGHSON RL: Adaptation of blood flow during the rest to work transition in humans. Med Sci Sports Exerc 31: 1019-1026, 1999. 
SHOEMAKER JK., TSCHAKOVSKY ME, HUGHSON RL: Vasodilation contributes to the rapid hyperemia with rhythmic contractions in humans. Can J Physiol Pharmacol 76: 418-427, 1998.

TSCHAKOVSKY ME, SHOEMAKER JK, HUGHSON RL: Vasodilation and muscle pump contribution to immediate exercise hyperemia. Am J Physiol 271: H1697-H1701, 1996.

TURNER DL: Cardiovascular and respiratory control mechanisms during exercise: an integrated view. J Exp Biol 160: 309-340, 1991. 